\title{
A MAXIMUM PRINCIPLE FOR OPTIMAL CONTROL PROBLEMS WITH FUNCTIONAL DIFFERENTIAL SYSTEMS ${ }^{1}$
}

\author{
BY H. T. BANKS
}

Communicated by Wolfgang Wasow, August 5, 1968

In this note we present a maximum principle in integral form for optimal control problems with delay-differential system equations which also contain delays in the control. Recent related results for particular cases of the systems discussed below may be found in [1], [5], and [6]. Vector matrix notation will be used and we shall not distinguish between a vector and its transpose.

Let $\alpha_{0}$ and $t_{0}$ be fixed in $R^{1}$ with $-\infty<\alpha_{0}<t_{0}, I=\left[\alpha_{0}, a\right)$ be a bounded interval containing $\left[\alpha_{0}, t_{0}\right]$, and put $I^{\prime}=\left(t_{0}, a\right)$. For $x$ continuous on $I$ and $t$ in $I^{\prime}$, the notation $F(x(\cdot), t)$ will mean that $F$ is a functional in $x$, depending on any or all of the values $x(\tau), \alpha_{0} \leqq \tau \leqq t$. $\bar{\Phi}$ will denote the class of absolutely continuous $n-1$ vector functions on $\left[\alpha_{0}, t_{0}\right]$. Let $\Omega$ be a given convex subset of the class of all bounded Borel measurable functions $u$ defined on $I$ into $R^{r}$, and $J$ be a given $C^{1}$ manifold in $R^{2 n-1}$. The problem considered is that of minimizing

$$
J\left[\bar{\phi}, u, \bar{x}, t_{1}\right]=\int_{t_{0}}^{t_{1}} f^{0}(\bar{x}(\cdot), u(\cdot), t) d t
$$

over $\bar{\Phi} \times \Omega \times C\left(I, R^{n-1}\right) \times I^{\prime}$ subject to

(i) $\quad \dot{\bar{x}}(t)=\bar{f}(\bar{x}(\cdot), u(\cdot), t)$

$$
\begin{array}{r}
\text { a.e. on }\left[t_{0}, t_{1}\right] \text {, } \\
\text { on }\left[\alpha_{0}, t_{0}\right] \text {, }
\end{array}
$$$$
\bar{x}(t)=\bar{\phi}(t)
$$

(ii) $\left(\bar{x}\left(t_{0}\right), \bar{x}\left(t_{1}\right), t_{1}\right) \in J$.

We assume that $f=\left(f^{0}, \bar{f}\right)=\left(f^{0}, f^{1}, \cdots, f^{n-1}\right)$ is an $n$-vector functional of the form

$$
\begin{aligned}
& f^{i}(\bar{x}(\cdot), u(\cdot), t)=h^{i}(\bar{x}(\cdot), t)+\int_{\alpha_{0}}^{t} u(s) d_{s} \eta(t, s) g^{i}(\bar{x}(s), t) \\
& \quad \text { for } i=0,1, \cdots, n-1,
\end{aligned}
$$

where the integral is a Lebesgue-Stieltjes integral. Each $h^{i}(\bar{x}(\cdot), t)$ is

1 This research was supported in part by the Air Force Office of Scientific Research, under Grant No. AF-AFOSR 693-67, in part by the National Aeronautics and Space Administration, under Grant No. NGR 40-002-015 and in part by the National Science Foundation, under Grant No. GP 9024. 
assumed $C^{1}$ in $\bar{x}$ and measurable in $t$, and each $g^{i}(\bar{y}, t)$ is $C^{1}$ in $(\bar{y}, t)$ on $R^{n}$. The $r \times 1$ vector function $\eta(t, s)$ is measurable in $t, s$, and of bounded variation in $s$ on $\left[\alpha_{0}, t\right]$. It is also assumed that the variation of $\eta$ is dominated by an $L_{1}\left(I^{\prime}\right)$ function $m$. That is, $V_{s=\alpha_{0}}^{t} \eta(t, s) \leqq m(t)$ for $t \in I^{\prime}$. Finally, suppose that given $\bar{X}$ compact, $\bar{X} \subset R^{n-1}$, there exists an $\tilde{m}$ in $L_{1}\left(I^{\prime}\right)$ such that $h=\left(h^{0}, h^{1}, \cdots, h^{n-1}\right)$ satisfies $|h(\bar{x}(\cdot), t)|$ $\leqq \tilde{m}(t)$ and $|d h[\bar{x}(\cdot), t ; \bar{\psi}]| \leqq \tilde{m}(t)\|\bar{\psi}\|_{t}$ for any $\bar{\psi} \in C\left(I, R^{n-1}\right)$ and $\bar{x} \in C(I, \bar{X})$, where $\|\bar{\psi}\|_{t}=\sup \left\{|\psi(s)|: s \in\left[\alpha_{0}, t\right]\right\}$ and $d h$ is the Fréchet derivative of $h$ with respect to $\bar{x}$. $(|A|$ denotes the Euclidean norm of $A$.)

If $\left(\Phi^{*}, u^{*}, \bar{x}^{*}, t_{1}^{*}\right)$ is a solution of the above problem, we define the $n \times n-1$ matrix function $\bar{\eta}^{*}$ for $t \in I^{\prime}, s \in\left[\alpha_{0}, t\right]$ by

$$
\bar{\eta}^{*}(t, s)=\bar{\eta}_{1}^{*}(t, s)+\bar{\eta}_{2}^{*}(t, s) .
$$

where $\bar{\eta}_{1}^{*}$ is such that

$$
d h\left[\bar{x}^{*}(\cdot), t ; \bar{\psi}\right]=\int_{\alpha_{0}}^{t} d_{s} \bar{\eta}_{1}^{*}(t, s) \bar{\psi}(s)
$$

for $t \in I^{\prime}$ and all $\Psi \in C\left(\left[\alpha_{0}, t\right], R^{n-1}\right)$, and

$$
\begin{aligned}
\bar{\eta}_{2}^{*}(t, s) & \equiv-\int_{0}^{t} u^{*}(\beta) d_{\beta} \eta(t, \beta) g_{\tilde{y}}\left(\bar{x}^{*}(\beta), t\right) & & s<t, \\
& \equiv 0 & & s \geqq t .
\end{aligned}
$$

(The existence of $\tilde{\eta}_{1}^{*}$ is guaranteed by the Riesz Theorem.) Then we have the following necessary conditions.

Theorem. Let $\left(\Phi^{*}, u^{*}, \bar{x}^{*}, t_{1}^{*}\right)$ be a solution to the problem under the assumptions above. In addition, suppose that $t_{1}^{*}$ is a Lebesgue point of $f\left(\bar{x}^{*}(\cdot), u^{*}(\cdot), t\right)$. Then there exists a nontrivial n-vector function $\lambda(t)=\left(\lambda^{0}(t), \bar{\lambda}(t)\right)$ of bounded variation on $\left[t_{0}, t_{1}^{*}\right]$, continuous at $t_{1}^{*}$, satisfying

(a) $\lambda^{0}(t)=$ constant $\leqq 0, \lambda\left(t_{1}^{*}\right) \neq 0$,

$$
\bar{\lambda}(t)+\int_{t}^{*} \lambda(\beta) \bar{\eta}^{*}(\beta, t) d \beta=\bar{\lambda}\left(t_{1}^{*}\right) \quad \text { for } t \in\left[t_{0}, t_{1}^{*}\right)
$$

where $\bar{\eta}^{*}$ is defined by (2).

(b)

$$
\int_{t_{0}}^{t_{1}^{*}} \lambda(t) f\left(\bar{x}^{*}(\cdot), u^{*}(\cdot), t\right) d t \geqq \int_{t_{0}}^{*} t_{1}^{*} \lambda(t) f\left(\bar{x}^{*}(\cdot), u(\cdot), t\right) d t
$$

for all $u \in \Omega$. 
(c) The $2 n-1$ vector

$$
\left(-\bar{\lambda}\left(t_{0}\right)+\int_{t_{0}}^{t_{1}^{*}} \lambda(\beta)\left\{\bar{\eta}^{*}\left(\beta, \alpha_{0}\right)-\bar{\eta}^{*}\left(\beta, t_{0}\right)\right\} d \beta, \bar{\lambda}\left(t_{1}^{*}\right),-\lambda\left(t_{1}^{*}\right) \cdot f^{*}\left(t_{1}^{*}\right)\right)
$$

is orthogonal to $J$ at $\left(\bar{x}^{*}\left(t_{0}\right), \bar{x}^{*}\left(t_{1}^{*}\right), t_{1}^{*}\right)$, where $f^{*}\left(t_{1}^{*}\right) \equiv f\left(\bar{x}^{*}(\cdot), u^{*}(\cdot), t_{1}^{*}\right)$.

The proof of this theorem involves showing that the class of functions $\mathcal{F}=\{F(\bar{x}(\cdot), t): F(\bar{x}(\cdot), t)=f(\bar{x}(\cdot), u(\cdot), t), u \in \Omega\}$ is absolutely quasiconvex [2] and then using necessary conditions for extremals given in [2]. Absolute quasiconvexity is a generalization of ideas due to Gamkrelidze [4], who first obtained an integral maximum principle for control problems with ordinary differential system equations. The inequality in (b) is a maximum principle in integral form for the above described optimal control problem.

In many particular cases of the systems defined by (1), one can show that the multipliers $\bar{\lambda}$ are actually absolutely continuous and satisfy (a) in differentiated form. This differentiated form becomes the usual known multiplier equation for systems with simple time lags in the state variables (see [1]). The transversality conditions given in (c) also can be reduced to a simpler form for many special cases of (1).

Included in (1) are many integro-differential systems and time lag systems which appear in physical problems. For example, if one modifies slightly the biological population model formulated by Cooke in [3], one obtains the system equation

$$
\dot{x}(t)=u(t-\tau) x(t-\tau)+\beta(t) u(t-\tau-\theta(t)) x(t-\tau-\theta(t))
$$

where $x(t)$ is the number in the population at time $t, u(t)$ is the conception rate at time $t$, and $\tau$ is the gestation period. Systems with

$$
h(\bar{x}(\cdot), t)=\int_{\alpha_{0}}^{t} A(t, s) q(\bar{x}(s), t) d s,
$$

which arise in the study of reactor dynamics [7], and with $h(\bar{x}(\cdot), t)$ $=G\left(\bar{x}_{t}, t\right)$, where $\bar{x}_{t}=\bar{x}(t+\theta), \theta \in[-T, 0]$, are also special cases of $(1)$.

\section{REFERENCES}

1. H. T. Banks, Necessary conditions for control problems with variable time lags J. SIAM Control 6 (1968), 9-47.

2. - Variational problems involving functional differential equations, J. SIAM Control 7 (1969).

3. K. L. Cooke, Functional-differential equations: Some models and perturbation problems, differential equations and dynamical systems, Academic Press, New York, 1967. 
4. R. V. Gamkrelidze, On some extremal problems in the theory of differential equations with applications to the theory of optimal control, J. SIAM Control 3 (1965), 106128.

5. E. B. Lee, Geometric theory of linear controlled systems (to appear).

6. - Variational problems for systems having delay in the conirol action (to appear).

7. J. J. Levin and J. A. Nohel, $A$ system of nonlinear integro-differential equations, Michigan Math. J. 13 (1966), 257-270.

Center for Dynamical Systems, Brown University, Providence, Rhode ISLAND 02904 